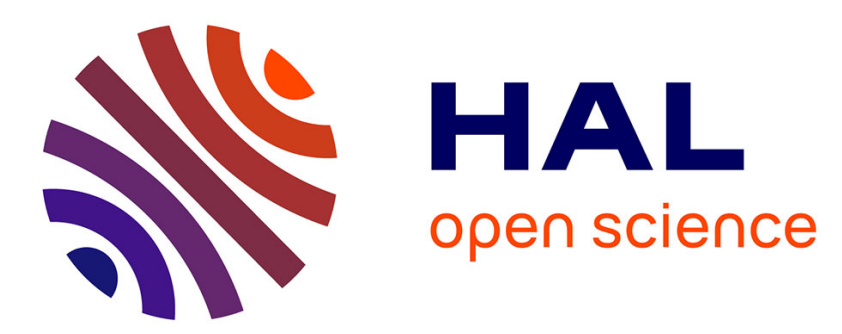

\title{
Récents développements sur la caractérisation des matériaux non cristallins par des méthodes de spectroscopie optique
}

\author{
G. Boulon
}

\section{- To cite this version:}

G. Boulon. Récents développements sur la caractérisation des matériaux non cristallins par des méthodes de spectroscopie optique. Revue de Physique Appliquée, 1977, 12 (5), pp.739-751. 10.1051/rphysap:01977001205073900 . jpa-00244235

\section{HAL Id: jpa-00244235 https://hal.science/jpa-00244235}

Submitted on 1 Jan 1977

HAL is a multi-disciplinary open access archive for the deposit and dissemination of scientific research documents, whether they are published or not. The documents may come from teaching and research institutions in France or abroad, or from public or private research centers.
L'archive ouverte pluridisciplinaire $\mathbf{H A L}$, est destinée au dépôt et à la diffusion de documents scientifiques de niveau recherche, publiés ou non, émanant des établissements d'enseignement et de recherche français ou étrangers, des laboratoires publics ou privés. 


\title{
RÉCENTS DÉVELOPPEMENTS SUR LA CARACTÉRISATION DES MATÉRIAUX NON CRISTALLINS PAR DES MÉTHODES DE SPECTROSCOPIE OPTIQUE
}

\author{
G. BOULON
}

E. R. No 10 du CNRS, Laboratoire de Spectroscopie et de Luminescence Université Claude-Bernard, Lyon I, 43, Bd du 11-Novembre-1918, 69621 Villeurbanne, France

Résumé. - Nous avons développé deux techniques de spectroscopie optique utilisées pour caractériser certaines propriétés des matériaux non cristallins :

1) La spectroscopie de diffusion Raman-laser, outil indispensable pour les recherches structurales. Nous développons comment on passe de l'interprétation des spectres des cristaux à ceux donnés par les matériaux non cristallins.

2) L'analyse de la photoluminescence des ions lourds $\left(\mathrm{Tl}^{+}, \mathrm{Pb}^{2+}, \mathrm{Bi}^{3+}\right)$ et des terres rares $\left(\mathrm{Eu}^{3+}\right.$, $\left.\mathrm{Ce}^{3+}, \ldots\right)$ par l'enregistrement des spectres d'excitation, d'émission et des déclins de fluorescence. On évalue la covalence de la liaison par la mesure du paramètre néphélauxétique de Jørgensen et les résultats spectroscopiques de la configuration $4 \mathrm{f}^{n}$ des terres rares donnent des informations sur l'ordre à courte distance, le nombre, la symétrie et la coordinence des sites occupés.

\begin{abstract}
We have developed two optical spectroscopy technics used to characterize some properties of non-cristalline materials :

1) Raman-laser spectroscopy, essential tool for structural researchs. We try to show how we can interpret Raman spectra both in cristalline and non-cristalline materials.

2) Analysis of the heavy ions $\left(\mathrm{Tl}^{+}, \mathrm{Pb}^{2+}, \mathrm{Bi}^{3+}\right)$ and rare-earth ions $\left(\mathrm{Eu}^{3+}, \mathrm{Ce}^{3+}, \ldots\right)$ photoluminescence in glasses from excitation, emission and fluorescence decay. We can measure covalency between the ligands and the central ion by nephelauxetic effect and also, with rare-earth ions, we can give some results about the short distance order, number, symmetry and coordination in occuped sites.
\end{abstract}

1. Introduction. - Ces dernières années de nombreuses études expérimentales ont été amorcées sur les verres dans le but de mieux connaître les propriétés chimiques et physiques indispensables pour la maitrise des applications qui s'avèrent d'ailleurs prometteuses. Nous nous sommes proposés lors de cette conférence de présenter quelques techniques de spectroscopie optique (domaines ultra-violet, visible et infra-rouge) utilisées pour caractériser ces matériaux : la diffusion Raman-laser et la photoluminescence des ions activateurs utilisés comme sonde. Nous avons illustré ces techniques par plusieurs exemples typiques portant sur des travaux récents. Dans ce qui suit, nous n'indiquerons que les grandes lignes présentées oralement dont on trouvera les explications détaillées dans les références citées. La démarche qui nous a paru la plus pédagogique est celle qui consiste à présenter quelques propriétés caractéristiques des cristaux puis de les comparer avec celles obtenues sur des matériaux non-cristallins donc plus désordonnés par rapport aux premiers. En effet, on considère généralement que ces matériaux qualifiés d'amorphes ou de verres ou non cristallins ou vitreux ne présentent un ordre qu'à courte distance c'est-à-dire dans un domaine incluant trois à quatre liaisons atomiques. On dit que ce désordre est topologique. Les corrélations à plus grande distance ne sont observables que dans les cristaux.

2. Spectroscopie Raman. Laser. - Ce n'est que depuis 1970 que l'on voit apparaître les premiers articles sur l'application de cette technique dans les verres. On se rapportera aux articles de base comme ceux de Bell [1], Dean [2] et Brawer [3] pour les verres d'oxydes, de Lucovsky [4] et Brodsky [5] sur les semi-conducteurs amorphes et Böttger [6] pour les solides non cristallins.

2.1 Présentation théoriQue. - C'est à partir de la notion de vibrations d'une chaîne diatomique infinie unidimensionnelle selon le modèle de Born et Von Karmann qu'il est aisé de définir les courbes de dispersion des modes longitudinaux et transverses à la fois pour les branches acoustiques et optiques de la première zone de Brillouin [7-8]. En fait, on applique le raisonnement précédent à un réseau tridimensionnel et la résolution des équations du mouvement des atomes est beaucoup plus complexe. Il est, néan- 
moins, possible d'évaluer les profils généraux des courbes de dispersion, pour une direction donnée du vecteur d'onde $\mathbf{K}$.

Pour un cristal moléculaire dont la maille élémentaire contient $n$ molécules constituées chacune de $N$ atomes, on a $3 \mathrm{Nn}$ modes de vibrations possibles pour chaque valeur de $K$. Parmi eux $n(3 N-6)$ sont dus aux vibrations internes de la molécule. Les $6 n$ degrés de liberté restants sont issus des 3 mouvements de rotation et des 3 mouvements de translation des molécules (Fig. 1) : ce sont les vibrations de réseau ou vibrations externes.

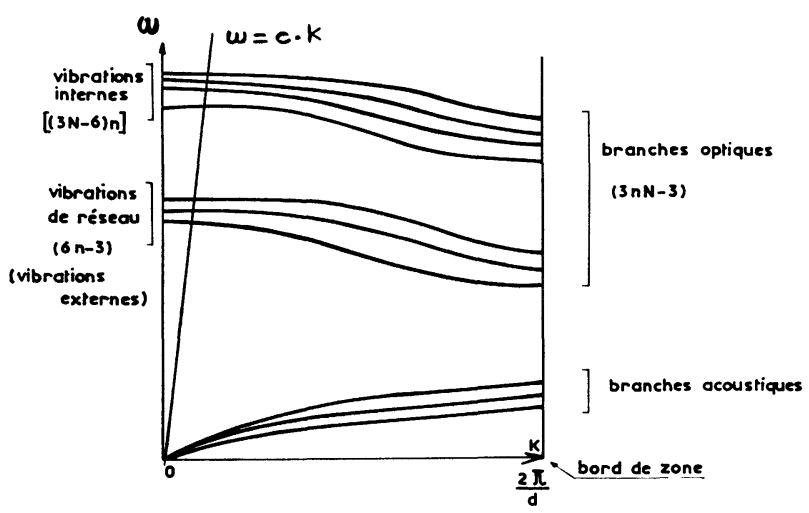

Fig. 1. - Allure générale des courbes de dispersion d'un cristal moléculaire pour une direction donnée de $K$ (première zone de Brillouin). La droite $\omega=c . K(c=$ célérité de la radiation excitatrice dans le cristal dont la valeur est environ $10^{5}$ fois celle du son dans le cristal) ne coupe que les branches optiques au voisinage de $K=0$.

La quantification des équations de mouvement permet d'obtenir $3 \mathrm{Nn}$ équations d'oscillateurs harmoniques indépendantes si les forces sont élastiques. Les fréquences caractéristiques sont celles des modes normaux de vibrations et la valeur propre de l'énergie de chaque oscillateur est $E_{n}=h v_{n}\left(v_{n}+\frac{1}{2}\right)\left(v_{n}=1,2 \ldots\right)$.

L'énergie totale est la somme des énergies de tous les oscillateurs. Les vibrations du réseau sont donc analogues à un système de particules possédant $3 n N$ niveaux d'énergie $h v_{n}$. Il peut y avoir un nombre $n$ quelconque de particules à chaque niveau et l'énergie totale du système est la somme d'une énergie de point zéro $\frac{1}{2} h v_{n}$ et de l'énergie de toutes les particules présentes. Ces particules sont les phonons ou particules associées aux ondes de vibrations du réseau d'énergie $h v_{\mathbf{n}}$ et de quantité de mouvement $\mathbf{p}=h \mathbf{K}$.

Comme les photons, les phonons possèdent deux propriétés fondamentales :

- sur un niveau d'énergie donné, leur nombre est quelconque. En conséquence, ils ne vérifient pas le principe d'exclusion de Pauli et suivent la statistique quantique de Bose-Einstein;

- l'excitation appropriée du cristal peut créer ou annihiler une de ces particules dont le nombre n'est déterminé que par les conditions spécifiques et plus particulièrement de sa température.
La détermination expérimentale des spectres de vibration est obtenue en excitant les vibrations par des rayonnements dont on étudie la diffusion. Les méthodes les plus connues sont basées sur la diffusion inélastique des photons $\mathrm{X}$, des photons visibles (diffusion Raman) et des neutrons thermiques. Les processus de diffusion inélastique peuvent être interprétés par collision entre les particules incidentes (photons ou neutrons) et les phonons. Les lois de conservation de l'énergie et de la quantité de mouvement entraînent :

$$
\begin{aligned}
\mathbf{K}_{\mathbf{i}} & =\mathbf{K}_{\mathrm{d}} \pm \mathbf{K} & & \text { (vecteurs d'onde) } \\
v_{\mathbf{i}} & =v_{\mathrm{d}} \pm v & & \text { (fréquences) }
\end{aligned}
$$

i : incident ; d : diffusé.

La relation de dispersion $v=f(\mathbf{K})$ est facilement déduite des mesures de $v_{\mathrm{d}}$ en fonction de $K_{\mathrm{d}}$ puisque $v_{\mathbf{i}}$ et $\mathbf{K}_{\mathbf{i}}$ sont connus.

Prenons l'exemple numérique suivant $\lambda_{\mathrm{j}}=5000 \AA$, $n$ (indice du milieu) $=1$,

$$
K_{\mathrm{i}}=\frac{2 \pi}{\lambda_{\mathrm{i}}} n \sim 10^{5} \mathrm{~cm}^{-1}
$$

Pour une géométrie de diffusion à $90^{\circ}$

$$
K \sim \sqrt{2 K_{\mathrm{i}}} \sim 10^{5} \mathrm{~cm}^{-1}
$$

La valeur maximum du vecteur d'onde des phonons $\mathbf{K}$ au bord de la zone de Brillouin est :

$$
K=\frac{\pi}{2 d} \sim 10^{8} \mathrm{~cm}^{-1} \text { pour } d=1 \AA \text {. }
$$

Donc, dans l'effet Raman du premier ordre les phonons observés seront $10^{3}$ fois plus petits que leur valeur maximum. Ils sont situés près du centre de la zone de Brillouin c'est-à-dire $K \sim 0$ et il n'y a pratiquement pas transfert de moment.

Par contre, il pourra y avoir transfert d'énergie puisque les fréquences des phonons sont inférieurs à $4000 \mathrm{~cm}^{-1}$ de sorte que $v_{\mathbf{i}} \sim v_{\mathrm{d}}$.

Ajoutons que les mêmes remarques s'appliquent à la spectroscopie infra-rouge $\left(\lambda_{\mathbf{i}}=10^{4}\right.$ à $\left.10^{7} \AA \gg d\right)$.

2.2 TECHNIQUe EXPÉRIMENTALE. - Tous les détails sont consignés dans l'article de Mooradian [9] où l'on trouvera en particulier les caractéristiques des principaux lasers utilisés ainsi que les modes opératoires pour éliminer la lumière diffusée parasite.

Enfin, rappelons que cette technique de diffusion Raman correspond à des fréquences de phonons supérieures à quelques dizaines $\mathrm{de} \mathrm{cm}^{-1}$ alors que la diffusion Brillouin, ou diffusion par les phonons acoustiques, analyse les spectres entre quelques dizièmes de $\mathrm{cm}^{-1}$ et un $\mathrm{cm}^{-1}$. Quant au spectre Rayleigh dû à la diffusion thermique donc aux modes qui ne se propagent pas, il donne le profil de la raie excitatrice diffusée pour des valeurs de $v<10^{-4}$ à $10^{-5} \mathrm{~cm}^{-1}$. 
2.3 DÉNOMBREMENT DES VIBRATIONS POUR UN CRISTAL [10-11-12-13]. - 2.3.1 Nature des groupes. On sait que l'ensemble des opérations de symétrie d'un cristal $R$ (rotation, réflexion, inversion, rotationréflexion) et $T$ (translations) conduisent à définir 230 groupes d'espace $G_{\mathrm{E}}$. L'ensemble des translations forme un sous-groupe $G_{\mathrm{T}}$ invariant du groupe d'espace. Les groupes $G_{\mathrm{T}}$ ainsi formés définissent les 14 réseaux de Bravais.

Le groupe facteur est le groupe quotient $G_{\mathrm{F}}=G_{\mathrm{E}} / G_{\mathrm{T}}$ dont les éléments sont $R$. Il est isomorphe de l'un des 32 groupes ponctuels qui caractérisent les classes des cristaux.

Les éléments de symétrie d'un atome ou d'une molécule dans un cristal sont ceux du site qu'il occupe dans la maille. Ces éléments $R$ constituent le groupe de site $G_{\mathrm{S}}$ qui est nécessairement un sous-groupe du groupe facteur $G_{\mathrm{F}}$.

2.3.2 Méthodes de dénombrement des vibrations. $\mathrm{Au}$ cours des vibrations principales, tous les atomes congruents effectuent en phase le même mouvement : elles appartiennent à la représentation totalement symétrique du groupe des translations $G_{\mathrm{T}}$. Il en résulte qu'il suffit de connaître les mouvements des atomes de la maille primitive pour connaître les vibrations principales. Pour ce faire, il existe deux méthodes :

- l'une basée sur le groupe facteur $G_{\mathrm{F}}$

- l'autre sur le groupe de site $G_{\mathrm{S}}$.

2.3.3 Méthode du groupe de site. - Il est établi par l'expérience que les molécules conservent leur individualité à l'état solide. Il est donc très utile de pouvoir décrire les vibrations internes des molécules dans la maille à partir de celle de la molécule isolée. C'est-à-dire de pouvoir établir la corrélation entre le groupe facteur $G_{\mathrm{F}}$ et le groupe ponctuel de la molécule $G_{\mathrm{M}}$. Ceci peut être fait à travers le groupe de site $G_{\mathrm{S}}$ qui est sous-groupe à $G_{\mathrm{F}}$ et $G_{\mathrm{M}}$.

Le dénombrement des vibrations de la molécule isolée étant établi, la corrélation entre $G_{\mathbf{M}}$ et $G_{\mathbf{S}}$ traduit l'effet statique de la symétrie du site sur les propriétés des vibrations moléculaires. La corrélation entre $G_{\mathrm{S}}$ et $G_{\mathrm{F}}$ traduit l'effet dynamique du couplage vibrationnel entre les diverses molécules de la maille primitive. Les tables de corrélation sont bien connues des utilisateurs, elles donnent les décompositions des représentations irréductibles d'un groupe en représentations irréductibles de ses sous-groupes [11-14].

2.3.4 Règles de sélection. - Les activités infrarouge et Raman des vibrations principales dans les cristaux peuvent être déduites par simple examen des tableaux de caractères du groupe ponctuel isomorphe du groupe facteur.

Activité infra-rouge: un mode de vibration se transforme comme la composante $x, y, z$ du vecteur de translation puisque aussi composante du vecteur électrique [8-11].
Activité Raman : un mode de vibration coïncide ou se transforme comme les composantes des éléments des tenseurs de polarisabilités dérivés [8-11].

\subsection{COMPARAISON ENTRE LES SPECTROSCOPIES RAMAN} ET INFRA-ROUGE. - Les spectres Raman sont beaucoup plus simples que les spectres infra-rouge : le nombre de raies est souvent limité, ces raies sont assez bien résolues, d'intensité notable et, bien sûr, polarisées à cause des vibrations totalement symétriques. De plus, cette technique de spectroscopie vibrationnelle sépare naturellement, d'une part, les vibrations internes des atomes dans le réseau dues aux liaisons de nature covalente et, d'autre part, les vibrations externes du réseau dont la nature est plus ionique. Elle permet aussi de séparer les masses des atomes présents et ceci est très utile pour les milieux amorphes où l'on peut distinguer les cations formateurs et perturbateurs du réseau.

D'autres avantages de la diffusion Raman par rapport à la spectroscopie infra-rouge peuvent être signalés: mesure plus facile des basses fréquences, enregistrements dans le domaine des hautes températures plus aisés, absence d'influence de la présence de petites quantités d'eau, facilité de la préparation des échantillons puisque la spectroscopie infra-rouge nécessite soit des films minces, soit des suspensions, enfin renseignements sur la structure interne au matériau alors que les ondes infra-rouge ne sont absorbées que sur une épaisseur très fine.

L'inconvénient majeur de la diffusion Raman est l'absence de mesure d'intensité absolue.

On voit, en conséquence, que l'on peut espérer atteindre une grande quantité d'informations structurales par ce moyen : fréquences de vibrations, forces de liaisons, propriétés de symétrie des vibrations.

2.5 EXEMPLE DU MONOCRISTAL $\mathrm{Bi}_{4} \mathrm{Ge}_{3} \mathrm{O}_{12}$ [15] (Fig. 2). - Ce germanate est isomorphe de l'eulytine (silicate de bismuth) et cristallise dans le système cubique. L'arrangement est caractérisé par des groupements tétraédriques isolés $\mathrm{GeO}_{4}$ occupant des sites de symétrie $\mathrm{S}_{4}$ et formant autour des atomes de $\mathrm{Bi}$ un voisinage octaédrique déformé d'oxygène.

Le mode du réseau étant cubique centré, la maille primitive ne contient que deux motifs, soit $n=38$ atomes ce qui correspond à $3 n-3=111$ degrés de liberté vibratoires. Les positions de ces atomes dans la maille conduisent au dénombrement suivant en ce qui concerne les phonons optiques de vecteur d'onde $K=0$ :

$$
\begin{gathered}
\Gamma_{27}=4 \mathrm{~A}_{1}+5 \mathrm{~A}_{2}+9 \mathrm{E}+14 \mathrm{~F}_{1}+14 \mathrm{~F}_{2} \\
\mathrm{~A}_{1}, \mathrm{E}, \mathrm{F}_{2}: \text { actifs Raman } \\
\mathrm{F}_{2}: \text { actif infra-rouge }
\end{gathered}
$$

Les groupements $\mathrm{GeO}_{4}$ étant bien individualisés dans la maille, nous séparerons en première approxi- 


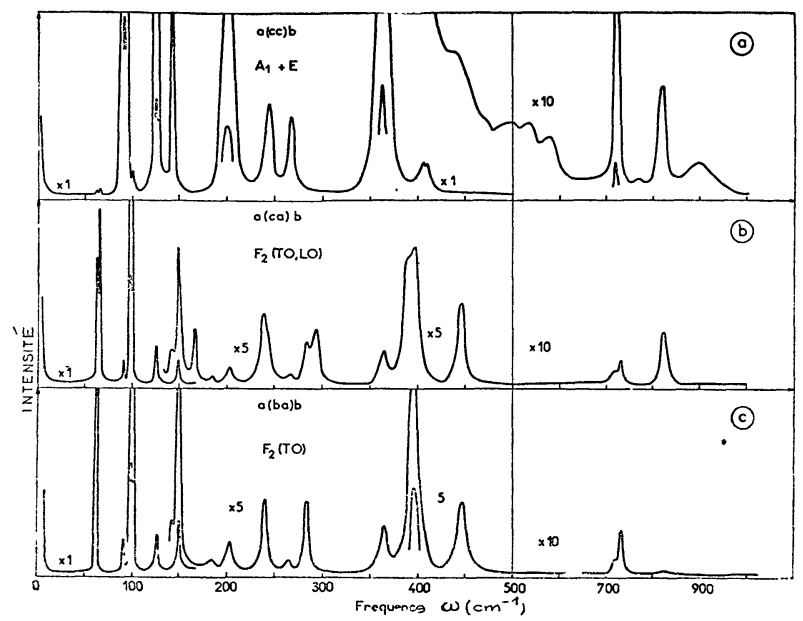

FIG. 2. - Spectres de diffusion Raman polarisés du monocristal $\mathrm{Bi}_{4} \mathrm{Ge}_{3} \mathrm{O}_{12}: a$ ) polarisation a(cc) $\mathrm{b}:$ modes de vibration $\left.\mathbf{A}_{1}+\mathbf{E} ; b\right)$ polarisation $\mathrm{a}(\mathrm{ca}) \mathrm{b}:$ modes de vibrations optiques $\mathrm{F}_{2}$ (longitudinaux LO et transverses $\mathrm{TO}$ ) ; $c$ ) polarisation $a(b a) b:$ modes de vibrations optiques transverses $F_{2}(T O)$. Noter le changement d'échelle à $500 \mathrm{~cm}^{-1}$.

mation les vibrations internes des tétraèdres des vibrations externes du réseau.

D'où les dénombrements partiels :

$$
\begin{aligned}
& \Gamma_{\mathrm{GeO}_{4}}=2 \mathrm{~A}_{1}+3 \mathrm{~A}_{2}+5 \mathrm{E}+6 \mathrm{~F}_{1}+7 \mathrm{~F}_{2} \\
& \Gamma_{\text {reseau }}=2 \mathrm{~A}_{1}+2 \mathrm{~A}_{2}+4 \mathrm{E}+8 \mathrm{~F}_{1}+7 \mathrm{~F}_{2} .
\end{aligned}
$$

Les corrélations entre les vibrations d'un tétraèdre isolé de symétrie $T_{\mathrm{d}}$ et celles des groupements $\mathrm{GeO}_{4}$ dans cette maille $T_{d}^{6}$ du germanate sont données sur le tableau I à partir des tables de caractères de $\mathrm{S}_{4}$ et $T_{d}$.

\section{TABLEAU I}

Corrélation entre les vibrations d'un tétraèdre isolé $\mathrm{GeO}_{4}$ et celles des groupes $\mathrm{GeO}_{4}$ dans la cellule unitaire $\mathrm{T}_{\mathrm{d}}^{6}$.

\begin{tabular}{|llll|}
\hline $\begin{array}{c}\mathrm{GeO}_{4} \text { isolé } \\
\mathrm{Td}\end{array}$ & $\begin{array}{c}\text { Cellule } \\
\text { unitaire } \\
\mathrm{Td}\end{array}$ & Activité \\
\hline$v_{1}$ & & Inactive \\
\hline$v_{3}, v_{4}$ & & Raman \\
\hline
\end{tabular}

$\left(^{*}\right) v_{1}$ et $v_{3}$ sont respectivement les fréquences de vibrations symétriques et dégénérées $v_{2}$ et $v_{4}$ sont les fréquences de vibrations dégénérées d'un tétraèdre $\mathrm{GeO}_{4}$ isolé.
Signalons que les modes polaires de type $F_{2}$ à la fois actifs en Raman et en infra-rouge peuvent éclater en composantes optiques transversales (TO) et longitudinales (LO).

Les attributions sont précisées sur le tableau II.

En conclusion les spectres infra-rouge et Raman du germanate de bismuth sont en bon accord avec le dénombrement théorique prévu à partir de la maille cubique $T_{d}$ puisque seulement deux modes de vibration sur les 27 attendus ne sont pas observés.

2.6 SPECTRES RAMAN DES MATÉRIAUX NON CRISTALLINS. - D'une manière générale, les spectres Raman des cristaux ne montrent que quelques raies fines dans le premier ordre en l'absence de centre d'inversion. C'est un résultat dû à la structure périodique du cristal puisque la fonction de corrélation défini par Shuker et Gamon [16] est périodique et que la transformée de Fourier est une fonction delta permettant la règle de sélection $K=0$. En conséquence, seuls quelques modes participent à la diffusion. Mais, dans les matériaux non cristallins, il n'y a pas de symétrie de translation et la fonction de corrélation apériodique peut être représentée par

$$
\mathrm{e}^{i \mathbf{K r}} \cdot \mathrm{e}^{-\boldsymbol{r} / \boldsymbol{\Lambda}}
$$

$\Lambda$ longueur de corrélation.

L'intervention du terme d'amortissement $\mathrm{e}^{-r / \Lambda}$ exclut la règle $K=0$ et tous les modes participent à la diffusion.

En sommant sur tous les modes de vibrations, Shuker et Gamon [16] calculent l'intensité des raies Stokes, de fréquence

$$
\begin{gathered}
I_{\text {diffusée }}(\omega)=\sum_{b} C_{b}^{\alpha, \beta, \gamma, \lambda}\left(\frac{1}{\omega}\right)[1+n(\omega, T)] \cdot g_{b}(\omega) \\
n(\omega, T)=\frac{1}{e \frac{\hbar \omega}{k T}-1}
\end{gathered}
$$

est la distribution de Bose-Einstein pour le premier ordre.

Chaque bande des états vibrationnels $b$ est supposée, pour des raisons de simplicité, avoir une constante de couplage $C_{b}^{\alpha, \beta, \gamma, \lambda}$ avec les indices $(\alpha, \beta)$ pour la polarisation de l'onde incidente $(\gamma, \lambda)$ pour celle de l'onde diffusée.

$g_{b}(\omega)$ est la densité d'états vibrationnels.

Pour une géométrie de polarisation donnée on déduit le spectre Raman réduit par :

$$
I_{\mathrm{R}}(\omega)=\sum_{b} C_{b}^{\alpha, \beta, \gamma, \lambda} g_{b}(\omega) .
$$

Celui-ci comprend à la fois la densité d'état et les éléments de matrices dont la variation avec $\omega$ est supposée faible par de nombreux auteurs.

En ce qui concerne les raies anti-Stokes, leur intensité est proportionnelle à $n(\omega, T)$, et non pas $n(\omega, T)+1$, ce qui explique qu'elles disparaissent aux basses températures. 
TABLEAU II

Mesures des fréquences infra-rouge et Raman de $\mathrm{Bi}_{4} \mathrm{Ge}_{3} \mathrm{O}_{12}$ et attributions possibles

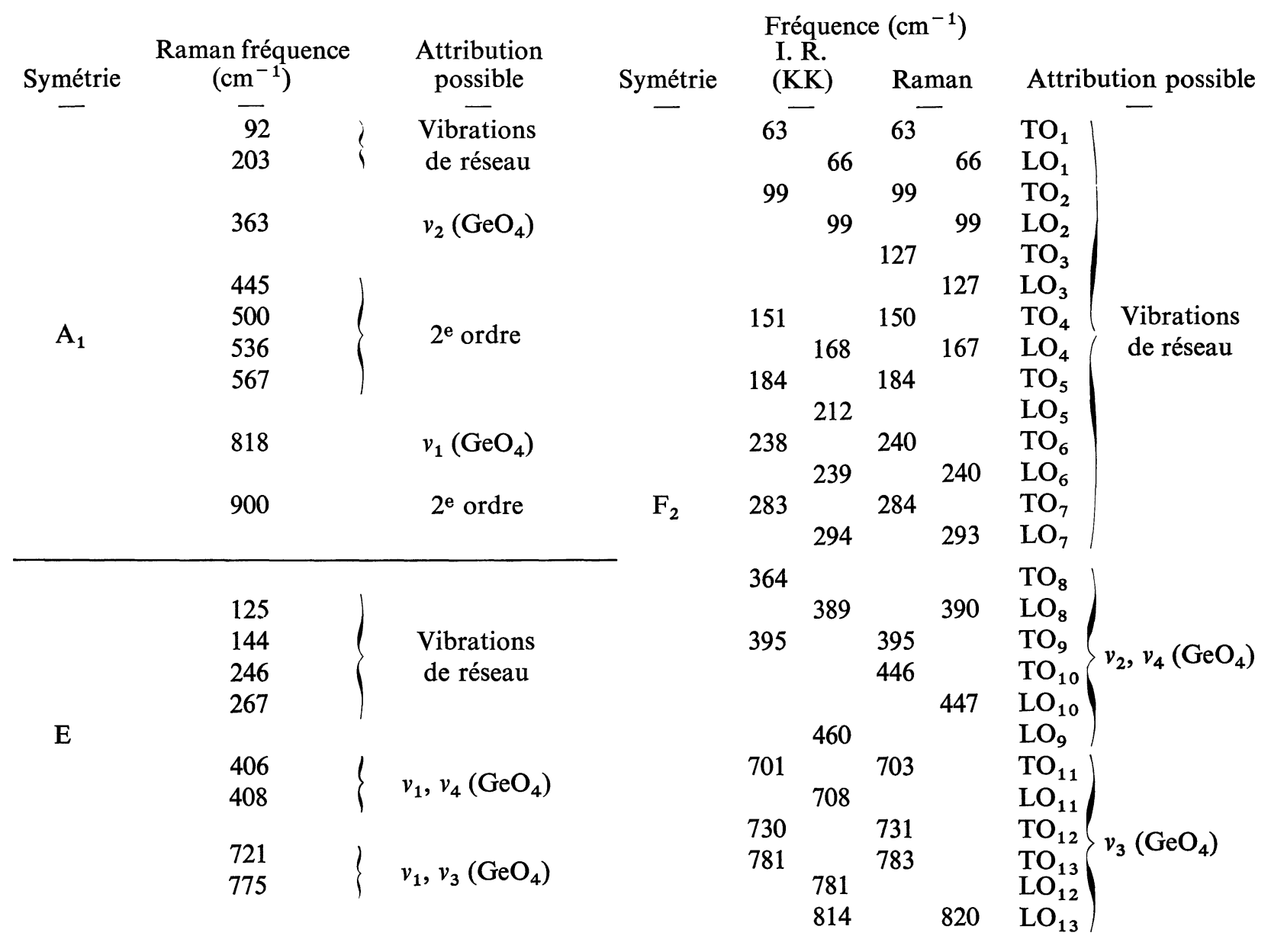

Il faut bien remarquer que la distribution de BoseEinstein n'est valable que pour les spectres du premier ordre et la population thermique diffère pour les autres ordres, cette distribution ayant une influence prépondérante sur le profil des spectres à haute température.

On trouvera de nombreux exemples de spectres Raman dans les références [9-16-17-18]. Lors de cette conférence nous avons comparé les densités d'états déterminées à partir des spectres infra-rouge, Raman et aussi par diffusion de neutrons. Les études les plus poussées sont celles sur les solides amorphes contenant des groupements tétraédriques $\left(\mathrm{SiO}_{4}, \mathrm{GeO}_{4}, \ldots\right)$. La figure 3 montre par exemple le spectre Raman polarisé du silicate de plomb et la figure 4 la densité d'état $g(\omega)$ déduite par Shuker et Gamon [19]. On note deux coupures vers 140 et $500 \mathrm{~cm}^{-1}$, absentes dans les silicates de verre, qui pourraient être associées à des microstructures. Shuker et Gamon attribuent le pic vers $40 \mathrm{~cm}^{-1}$ (Fig. 3) à la population thermique à $300 \mathrm{~K}$ ce qui montre qu'il faut être particulièrement prudent lors des attributions de ces spectres.

Les spectres Raman sont aussi utilisés pour caractériser certains matériaux durant leur cristallisation. C'est le cas de $\mathrm{As}_{2} \mathrm{~S}_{3}$ à $465 \mathrm{~K}$ [5]. Pour ce composé

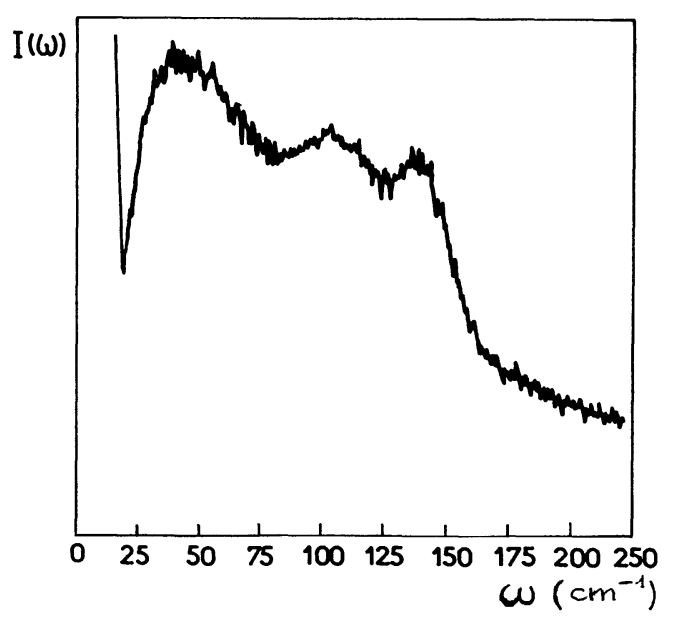

FIG. 3. - Spectre de diffusion Raman polarisé du silicate de plomb $\left(\mathrm{Pb} / \mathrm{SiO}_{2}\right)$ pour les basses fréquences. L'unité de l'intensité $I(\omega)$ est arbitraire. La fréquence $\omega$ est représentée en $\mathrm{cm}^{-1}$. D'après [19].

il convient d'être soigneux dans l'analyse spectrale en raison de l'intervention des modes de couches rigides pour des fréquences inférieures à $75 \mathrm{~cm}^{-1}$. Des mesures à haute température $(1040 \mathrm{~K})$ ont été 


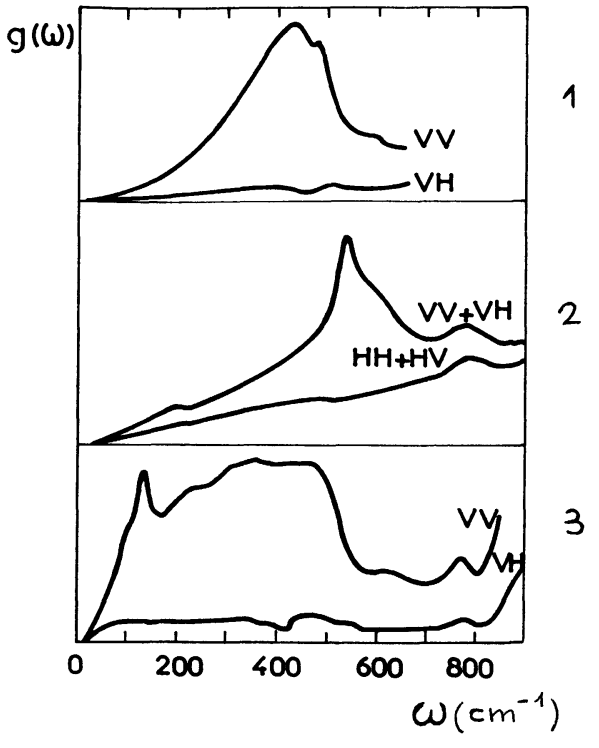

Fig. 4. - Densité d'états $g(\omega)$ de plusieurs matériaux vitreux calculée selon la relation $I_{\mathrm{R}}(\omega)$ du paragraphe 2.6.1) $\mathrm{Ti} / \mathrm{SiO}_{2}$; 2) $\mathrm{Na} / \mathrm{SiO}_{2}$; 3) $\mathrm{Pb} / \mathrm{SiO}_{2}$. D'après [19].

publiées par Finkman : même dans le sel fondu $\mathrm{As}_{2} \mathrm{~S}_{3}$, les spectres Raman sont identiques. Ce serait l'évidence d'une structure, en couche avec des vibrations d'unités pyramidales $\mathrm{AsS}_{3}$ et de molécules $\mathrm{As}_{2} \mathrm{~S}$ du type $\mathrm{H}_{2} \mathrm{O}$ à l'intérieur de ces couches [20].

Nous avons aussi montré la caractérisation typique des trois formes (cristal-trigonal, cristal-monoclinique et composé amorphe) du sélénium [21]. Les pics du composé amorphe semblent indiquer le mélange de chaînes et d'anneaux dans un rapport qui n'a pas pu être défini.

2.7 ESSAI D'ANALYSE THÉORIQUE DES VERRES OXYGÉNÉs. - Plusieurs chercheurs comme Gaskell, Shuker, Gamon, Bell, etc... ont essayé de donner une description fondamentale des spectres vibrationnels des verres. La plus récente description et, semble-t-il, l'une des meilleures, est celle de Brawer [3].

Brawer considère les réseaux vitreux oxygénés constitués de plusieurs groupes structuraux formés par plusieurs atomes. Cet auteur compare les groupes structuraux avec les cellules unitaires d'un cristal. Les équations de mouvement d'un groupe structural inclut le désordre. Le désordre signifie ici la déviation à partir d'une répétition strictement translationnelle des groupes, laquelle pourrait convertir le verre à l'état cristallin. Le résultat de ces équations dépend du couplage des modes des groupes structuraux.

L'intensité Raman diffusée et la largeur des pics sont étudiées plus particulièrement dans deux cas pour lesquels l'interaction entre les groupes structuraux est d'une part, négligeable (structure moléculaire) et, d'autre part, notable.

Les principales conclusions sont :

- Les spectres des verres et des cristaux qui ont la même composition montrent un excellent accord si certaines conditions sont satisfaites : les groupes structuraux doivent être présents à la fois dans le cristal et le verre et il doit $\mathrm{y}$ avoir très peu de couplage entre ces groupes. Ce couplage doit d'ailleurs être considéré pour chaque mode vibrationnel. L'accord demeure si le degré de désordre croît mais la largeur des pics augmente avec l'accroissement du désordre.

- Le désordre dans les verres peut augmenter la polarisabilité. Pour cette raison certains modes qui n'étaient pas visibles dans le cristal peuvent devenir visible dans le spectre du verre correspondant.

- Dans le cas d'un fort couplage, les calculs montrent que les largeurs des modes croissent d'une façon considérable.

Cette méthode de Brawer vient d'être appliquée par Bril sur des borates vitreux et cristallins [22].

3. Photoluminescence de matériaux activés. 3.1 Présentation GÉNÉrale. - Nous avons développé dans cette deuxième partie quelques informations tirées des propriétés liées à la photoluminescence des ions activateurs dans les verres. Les résultats expérimentaux sont, dans ce cas, les spectres d'absorption, d'excitation, de fluorescence et les déclins de fluorescence. Les ouvrages de base de D. Curie [23-24], Di Bartolo [8] et P. Caro [25] fournissent les explications fondamentales sur l'utilisation de cette technique spectroscopique. Depuis quelques années, de nombreux articles paraissent sur les exploitations des spectres obtenus soit avec des ions lourds $\left(\mathrm{Tl}^{+}, \mathrm{Pb}^{2+}\right.$, $\left.\mathrm{Bi}^{3+}\right)$ soit avec des ions terres rares $\left(\mathrm{Ce}^{3+}, \mathrm{Nd}^{3+}\right.$, $\mathrm{Eu}^{3+}, \mathrm{Tb}^{3+}$...). Ces recherches ont d'ailleurs, dans de nombreux cas, des objectifs d'application des matériaux vitreux comme milieux lasers et milieux standards de fluorescence [26]. Les travaux les plus importants sont menés par Duffy et Ingram [27], Reisfeld et coll. [26-28-29-30-31-32-33] ainsi que par Weber et Riseberg [34-35-36].

Nous rappellerons simplement que lorsqu'un ion est introduit en substitution dans un réseau cristallin sa symétrie n'est plus sphérique comme pour l'ion libre, mais est celle du site ponctuel cristallographique occupé. La dégénérescence des niveaux est alors levée, par l'interaction de l'ion avec le réseau. Cet ion est soumis principalement à deux effets dus au champ cristallin créé par les charges électriques négatives des ions voisins dont l'ensemble définit la symétrie du site et au champ de ligandes, c'est-à-dire à l'effet de la liaison chimique entre l'ion activateur et les ions voisins pour des directions précises. La théorie des groupes permet de définir les propriétés de symétrie des fonctions d'onde de l'ion dans le cristal et chaque niveau peut être étiqueté par un symbole. Quant aux différences d'énergie entre les niveaux on peut les calculer soit à partir des calculs d'orbitales moléculaires par une méthode auto-cohérente sur la charge et la configuration (voir les travaux de l'ion $\mathbf{B i}^{3+}$ [37-38-39]), soit à partir du calcul, mieux connu pour les terres 
rares, de l'hamiltonien $H_{\mathrm{c}}$ de champ cristallin. Il est, en effet, aisé de retrouver cet hamiltonien sous la forme :

$$
H_{\mathrm{c}}=-e \sum_{i} V\left(r_{i}, \theta_{i}, \varphi_{i}\right),
$$

pour un activateur comprenant $i$ électrons entourés de $\mathrm{n}$ ions négatifs de charges $q_{n}$, aux points définis $\operatorname{par} R_{n}, \alpha_{n}, \beta_{n}$.

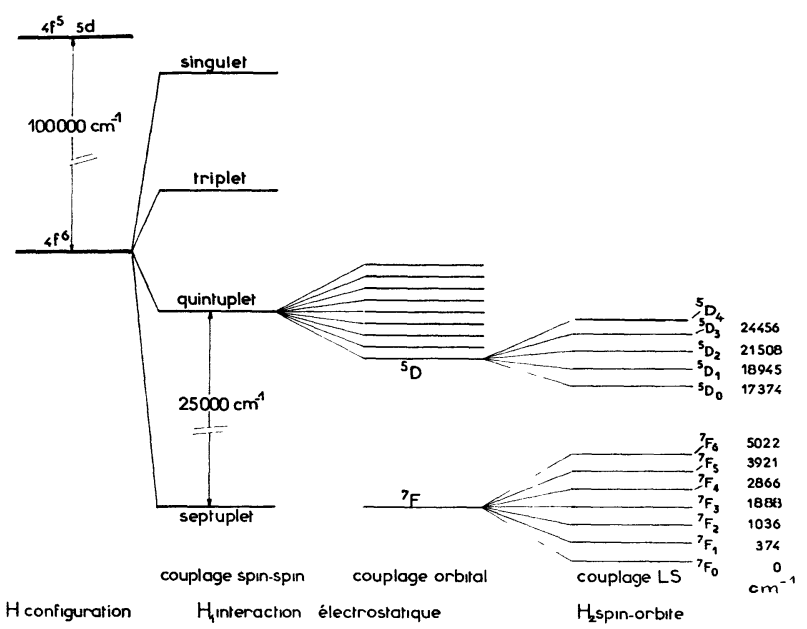

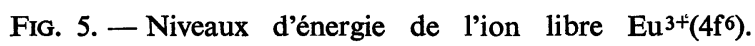

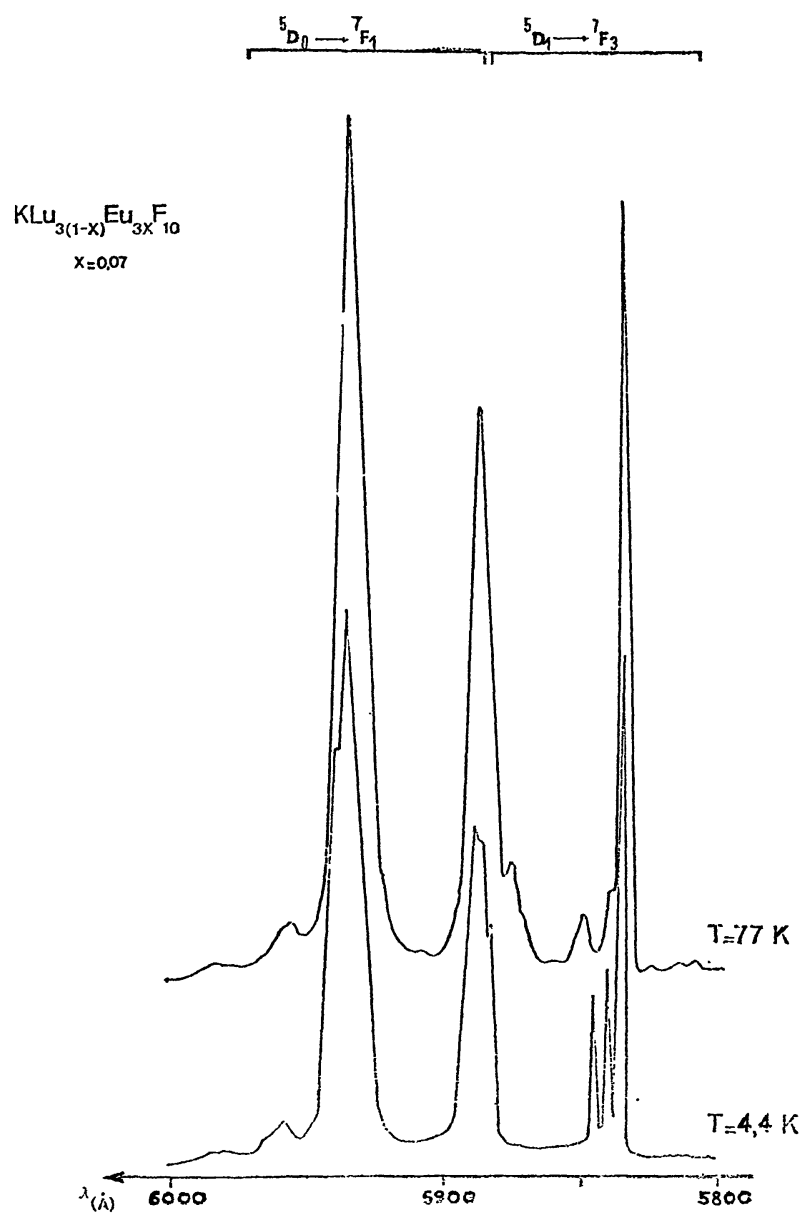

Fig. 6. - Spectres d'émission de $\mathrm{KLu}_{3} \mathrm{~F}_{10}\left(\mathrm{Eu}^{3+}\right)$ entre 5800 et $6000 \AA$ à 77 et $4,4 \mathrm{~K}$.
$V(r, \theta, \varphi)$ est le potentiel électrique au point $(r, \theta, \varphi)$ dû aux charges ponctuelles entourant le cation qui est solution de $\Delta V=0$. On obtient alors :

$$
\begin{aligned}
& H_{\mathrm{c}}=\sum_{i, k, q} A_{k}^{q} r_{i}^{k} Y_{k}^{q}\left(\theta_{i}, \psi_{i}\right) \\
& A_{k}^{q}=\frac{4 \pi}{2 k+1} \sum_{n} \frac{q_{n} e^{2}}{R_{n}^{k+1}}\left(Y_{k}^{q}\right)^{*}\left(\alpha_{n}, \beta_{n}\right)
\end{aligned}
$$

( $Y_{k}^{q}$ désigne l'harmonique sphérique).

On écrit $H_{\mathrm{c}}$ sous la forme de produits des coefficients $B_{q}^{k}$ par les opérateurs tensoriels $\left(C_{q}^{k}\right)_{i}$ :

$$
H_{\mathrm{c}}=\sum_{i, k, q} B_{q}^{k}\left(C_{q}^{k}\right)_{i}
$$

$k \leqslant \theta$ pour la configuration $f^{N}$ des terres rares.

Les coefficients $B_{q}^{k}$ sont considérés comme des paramètres phénoménologiques ajustables aux mesures expérimentales.

On sait que l'un des intérêts spectroscopiques des terres rares réside dans l'inégalité des hamiltoniens inter-électronique $H_{1}$, de couplage spin-orbite $H_{2}$ et de champ cristallin $H_{\mathrm{c}}: H_{1}>H_{2}>H_{\mathrm{c}}$ (Fig. 5). Si bien que le degré de mélange des états reste faible lorsque l'ion activateur est incorproé dans la matrice cristalline.

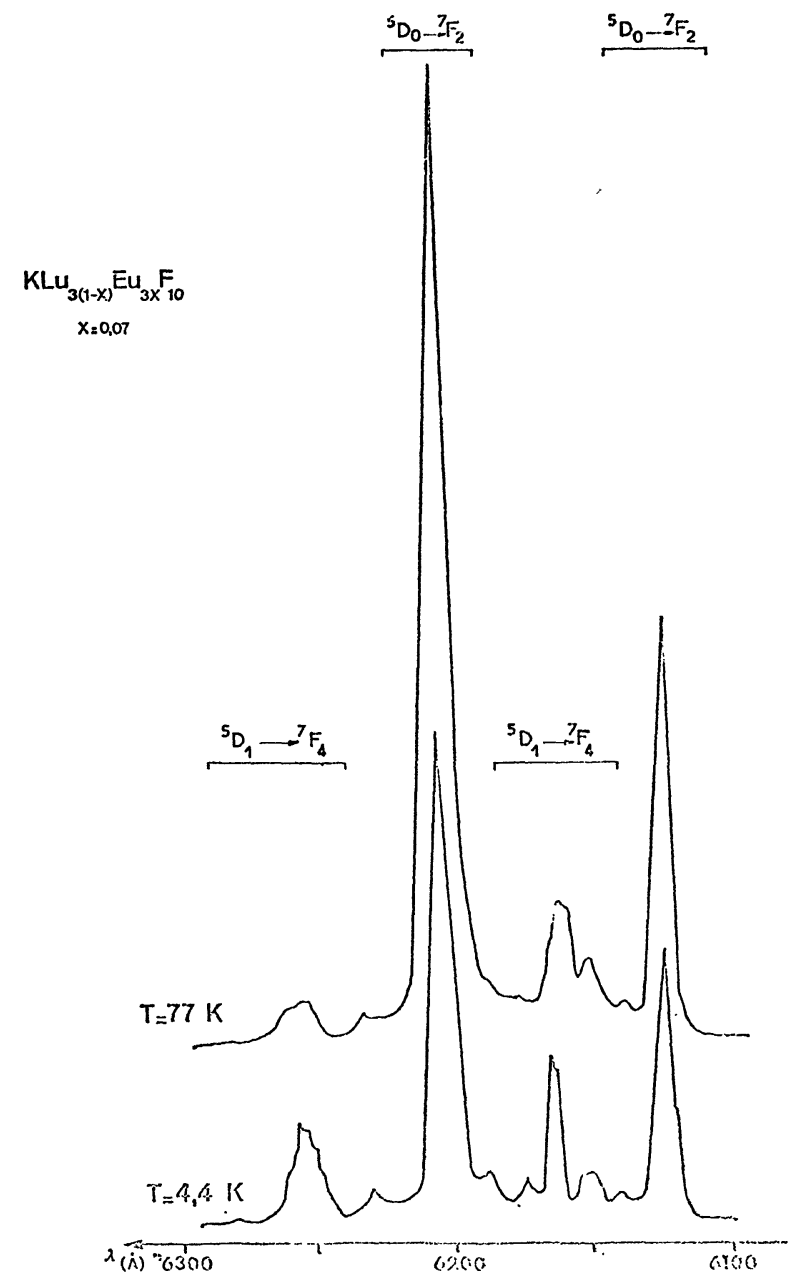

FIG. 7. - Spectres d'émission de $\mathrm{KLu}_{3} \mathrm{~F}_{10}\left(\mathrm{Eu}^{3+}\right)$ entre 6100 et $6300 \AA$ à 77 et $4,4 \mathrm{~K}$. 

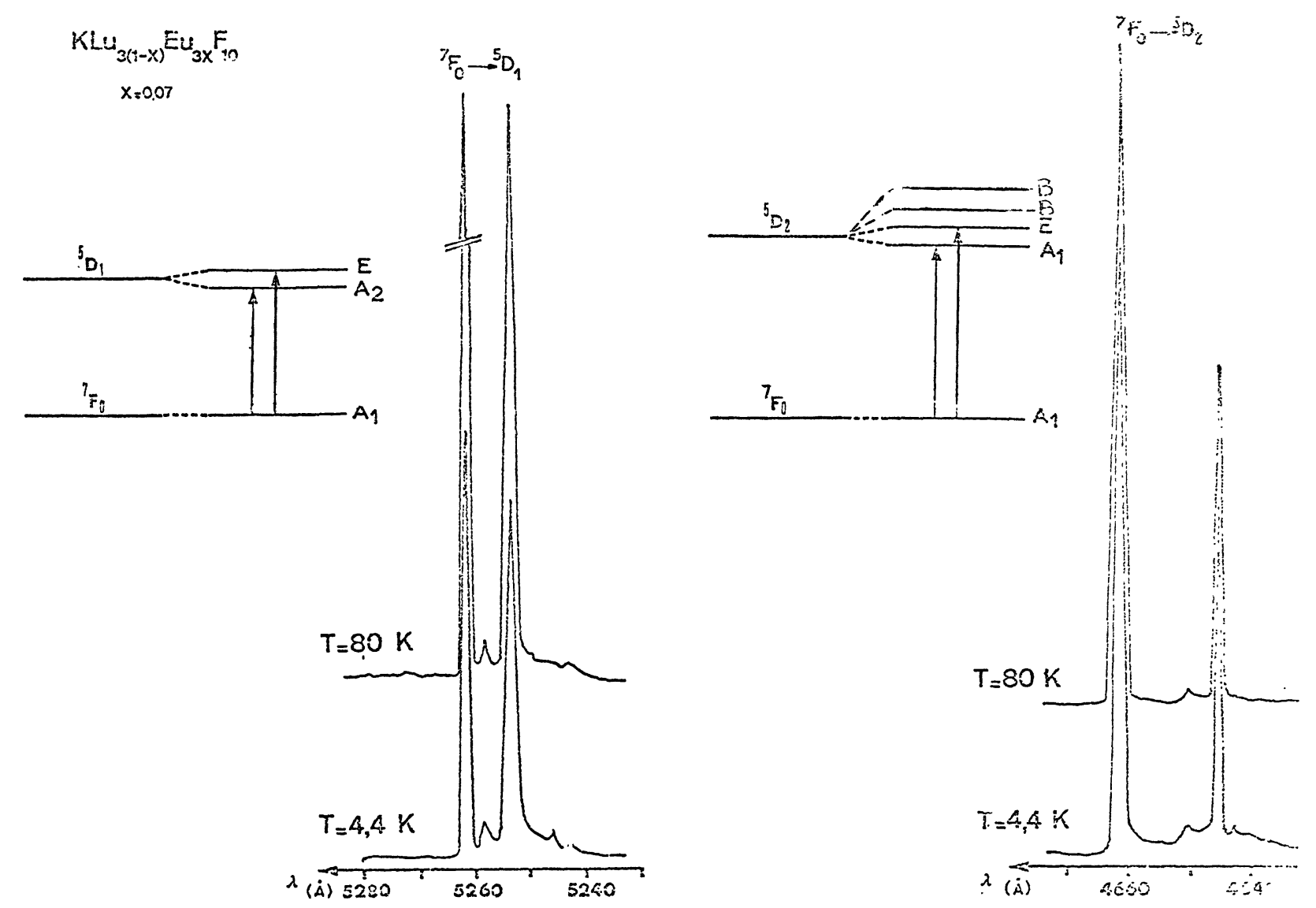

Fig. 8. - Spectres d'excitation de $\mathrm{KLu}_{3} \mathrm{~F}_{10}\left(\mathrm{Eu}^{3+}\right)$ à 80 et $4,4 \mathrm{~K}$ des niveaux ${ }^{5} \mathrm{D}_{1}$ et ${ }^{5} \mathrm{D}_{2}$ de l'ion $^{\text {Eu }}{ }^{3+}$.

Un niveau $J$ donné est décomposé selon $2 J+1$ composantes si $J$ pair et $J+\frac{1}{2}$ si $J$ impair selon la symétrie du site. Cette décomposition est traduite par une série de raies fines entre les niveaux de l'état fondamental et les divers niveaux des états excités en respectant les règles de sélection des transitions dipolaires électriques DE et dipolaires magnétiques DM propres à chacune des symétries. En présence d'un centre d'inversion l'intensité des DM est supérieure à celle des DE tandis qu'en l'absence de centre d'inversion l'inverse peut se produire. Ainsi, par exemple, pour $\mathrm{Eu}^{3+}{ }^{7} \mathrm{~F}_{0} \rightleftarrows{ }^{5} \mathrm{D}_{1},{ }^{5} \mathrm{D}_{0} \rightleftarrows{ }^{7} \mathrm{~F}_{1}$ sont $\mathrm{DM}$ et ${ }^{7} \mathrm{~F}_{0} \rightleftarrows{ }^{5} \mathrm{D}_{2}$, ${ }^{5} \mathrm{D}_{0} \rightleftarrows{ }^{7} \mathrm{~F}_{2}$ sont de nature DE. Quant à ${ }^{5} \mathrm{D}_{0} \rightleftarrows{ }^{7} \mathrm{~F}_{0}$ normalement interdite, la théorie des groupes prévoit sa présence en symétrie $C_{s}, C_{n}$ et $C_{n v}$ et c'est une transition $\mathrm{DE}$ qui ne peut être décomposée quelle que soit la symétrie cristallographique. L'évolution du nombre de raies de chaque transition et plus particulièrement de ${ }^{5} \mathrm{D}_{0} \rightleftarrows{ }^{7} \mathrm{~F}_{0}$ joue un rôle important quand on utilise $\mathrm{Eu}^{3+}$ comme sonde structurale. Les figures 6,7 et 8 représentent quelques raies de $\mathrm{Eu}^{3+}$ en substitution de $\mathrm{Lu}^{3+}$ dans $\mathrm{KLu}_{3} \mathrm{~F}_{10}$ [40]. Le nombre de composantes Starck est en bon accord avec la symétrie $\mathrm{C}_{4 \mathrm{v}}$ occupé par l'activateur. Les tableaux III.a, III.b permettent de se rendre compte du nombre de niveaux et de leurs représentations irréductibles ainsi que des règles de sélection pour $\mathrm{DE}$ et $\mathrm{DM}$ en symétrie $\mathrm{C}_{4 \mathrm{v}}$. Si l'on introduit cette terre rare dans une matrice

\section{TABLEAU III}

a) Nombre de niveaux en symétrie $C_{4 \mathrm{v}}$.

b) Règles de sélection pour les transitions dipolaires électriques D. E. et dipolaires magnétiques D. M. Nature de la polarisation ( $\sigma$ ou $\pi$ ) des transitions.

a)

\begin{tabular}{l|lllllll}
$\mathrm{J}$ & 0 & 1 & 2 & 3 & 4 & 5 & 6 \\
\hline $\mathrm{A}_{1}$ & 1 & 0 & 1 & 0 & 2 & 1 & 2 \\
$\mathrm{~A}_{2}$ & 0 & 1 & 0 & 1 & 1 & 2 & 1 \\
$\mathrm{~B}_{1}$ & 0 & 0 & 1 & 1 & 1 & 1 & 2 \\
$\mathrm{~B}_{2}$ & 0 & 0 & 1 & 1 & 1 & 1 & 2 \\
$\mathrm{E}$ & 0 & 1 & 1 & 2 & 2 & 3 & 3
\end{tabular}

b)

\begin{tabular}{l|lllll} 
& $\mathrm{A}_{1}$ & $\mathrm{~A}_{2}$ & $\mathrm{~B}_{1}$ & $\mathrm{~B}_{2}$ & $\mathrm{E}$ \\
\hline $\mathrm{A}_{1}$ & $\pi$ & & & & $\sigma$ \\
$\mathrm{A}_{2}$ & & $\pi$ & & & $\sigma$ \\
$\mathrm{B}_{1}$ & & & $\pi$ & & $\sigma$ \\
$\mathrm{B}_{2}$ & & & & $\pi$ & $\sigma$ \\
$\mathrm{E}$ & $\sigma$ & $\sigma$ & $\sigma$ & $\sigma$ & $\sigma$
\end{tabular}

D. E.

\begin{tabular}{l|lllll} 
& $\mathrm{A}_{1}$ & $\mathrm{~A}_{2}$ & $\mathrm{~B}_{1}$ & $\mathrm{~B}_{2}$ & $\mathrm{E}$ \\
\hline $\mathrm{A}_{1}$ & & $\sigma$ & & & $\pi$ \\
$\mathrm{A}_{2}$ & $\sigma$ & & & & $\pi$ \\
$\mathrm{B}_{1}$ & & & & $\sigma$ & $\pi$ \\
$\mathrm{B}_{2}$ & & & $\sigma$ & & $\pi$ \\
$\mathrm{E}$ & $\pi$ & $\pi$ & $\pi$ & $\pi$ & $\pi$
\end{tabular}

D. M. comme $\mathrm{BaCaLu}_{2} \mathrm{~F}_{10}$, elle peut occuper trois types de sites de substitution (Ba de symétrie Td, $\mathrm{Ca}$ et $\mathrm{Lu}$ de symétrie $\mathrm{C}_{4 \mathrm{v}}$ ) et il faut s'attendre à observer des raies 


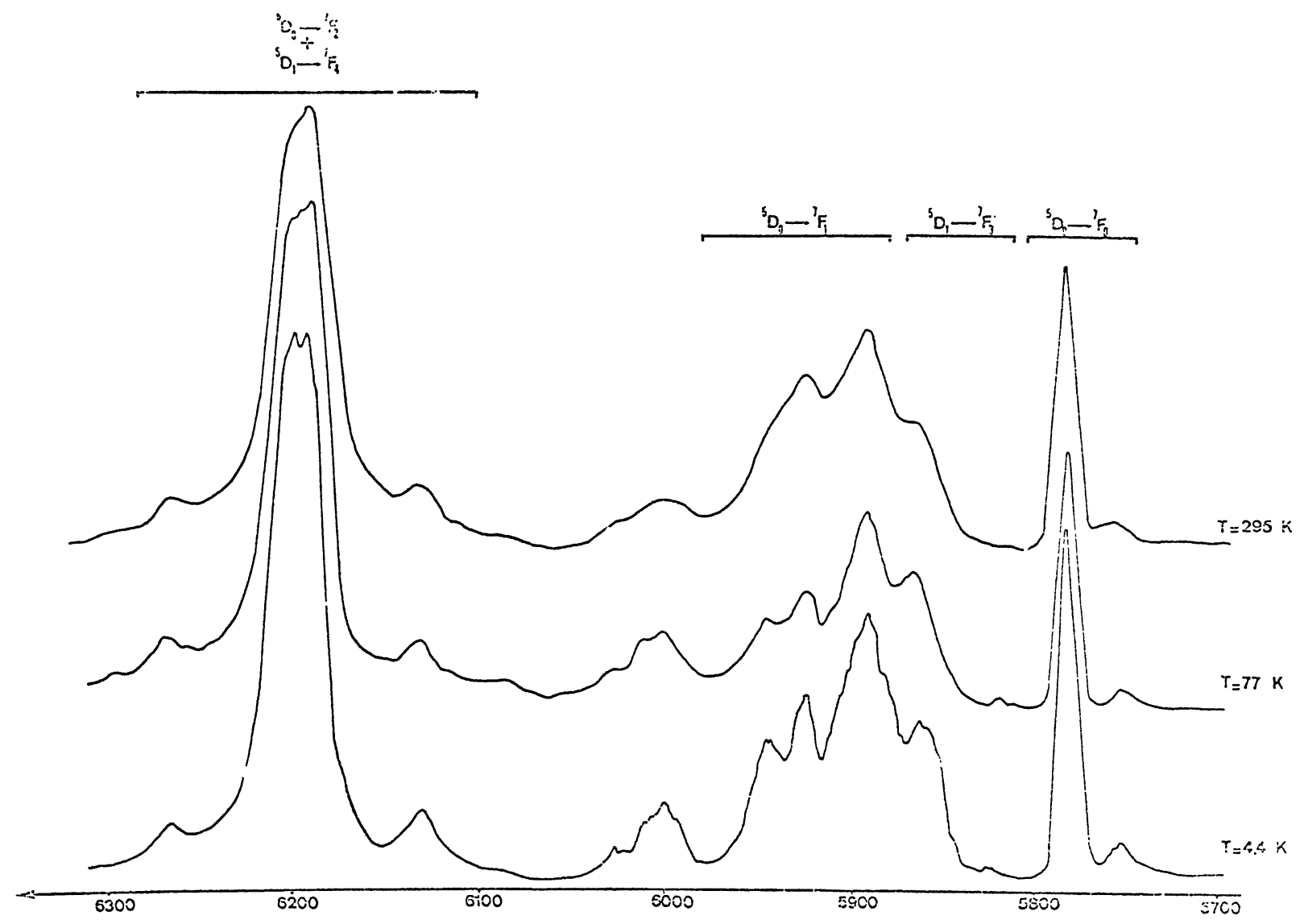

FIG. 9. - Spectres d'émission entre 5700 et $6300 \AA$ de $\mathrm{BaCaLu}_{2(1-x)} \mathrm{Eu}_{2 x} \mathrm{~F}_{10}(x=0,075)$ à 295,77 et 4,4 K. On reconnaît les transitions $5 D_{0} \rightarrow 7 F_{0}, 5 D_{1} \rightarrow 7 F_{3}, 5 D_{0} \rightarrow 7 F_{1}$ et ${ }^{5} D_{0} \rightarrow 7 F_{2}+5 D_{0} \rightarrow 7 F_{4}$.

beaucoup plus larges en raison du recouvrement spectral des raies voisines associées à chaque site. Sur la figure 9 , on voit que même à $4,4 \mathrm{~K}$ la résolution du spectre d'émission est médiocre. Mais l'information la

$$
\begin{aligned}
& { }^{7} \mathrm{~F}_{0}-{ }^{5} \mathrm{D}_{0} \\
& \mathrm{~T}=4,4 \mathrm{~K}
\end{aligned}
$$

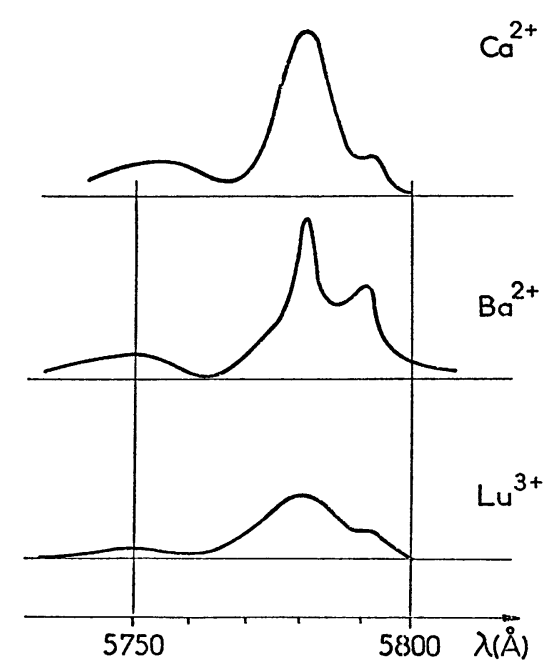

FIG. 10. - Spectres d'excitation de la transition $7 \mathbf{F}_{0} \rightarrow 5 \mathbf{D}_{0}$ à $4,4 \mathrm{~K}$ lorsque $\mathrm{Eu}^{3+}$ est substitué respectivement sur les sites $\mathrm{Ca}^{2+}, \mathrm{Ba}^{2+}$ et $\mathrm{Lu}^{3+}$. plus intéressante est donnée par l'apparition sur le spectre d'excitation (Fig. 10) de trois raies ${ }^{7} \mathrm{~F}_{0} \rightarrow{ }^{5} \mathrm{D}_{0}$ liées aux trois sites de symétrie (un Td déformé et deux $\mathrm{C}_{4 \mathrm{v}}$ ) [40]. Par conséquent, les propriétés spectrales de la photoluminescence de l'europium dans les verres doit dépendre fortement de la pluralité des sites dont la symétrie est souvent très basse. Le désordre introduit des effets perturbateurs réfléchis par les largeurs inhomogènes des raies de fluorescence. On comprend mieux pourquoi ce sont les terres rares qui sont des sondes ponctuelles (décomposition du champ cristallin de l'ordre de 100 à $300 \mathrm{~cm}^{-1}$ ) et non pas les ions de transition pour lesquels la décomposition est de plusieurs milliers de $\mathrm{cm}^{-1}$.

3.2 CaRactéRisation DU DEGRÉ DE COVALENCE DE LA LIAISON ET DE LA DISTANCE CRISTALLINE. - Il semble que les analyses les plus sérieuses soient celles portant sur des séries de verres dans lesquels on peut modifier la nature des cations du réseau formateur et celle des cations du réseau perturbateur comme, par exemple, les phosphates, borates et germanates de $\mathbf{K}$, $\mathrm{Mg}, \mathrm{Ca}, \mathrm{Sr}, \mathrm{Ba}$ [28]. On peut, dans ces conditions, suivre l'évolution et la valeur de la décomposition de certaines raies comme ${ }^{5} \mathrm{D}_{0} \rightarrow{ }^{7} \mathrm{~F}_{0}$ ou ${ }^{5} \mathrm{D}_{0} \rightarrow{ }^{7} \mathrm{~F}_{1}$ de la configuration $4 \mathrm{f}^{6}$ ainsi que des bandes larges ultraviolettes attribuées au transfert de charge $\mathrm{Eu}^{3+}-\mathrm{O}^{2-}$ ou au passage interconfigurationnel $4 \mathrm{f} \rightarrow 5 \mathrm{~d}$. Reisfeld 


\section{TABLEAU IV}

Energies des maximums des bandes d'excitations en milliers de $\mathrm{cm}^{-1}$ de $\mathrm{Eu}^{3+}, \mathrm{Tb}^{3+}$ et $\mathrm{Pb}^{2+}$ d'après [28]

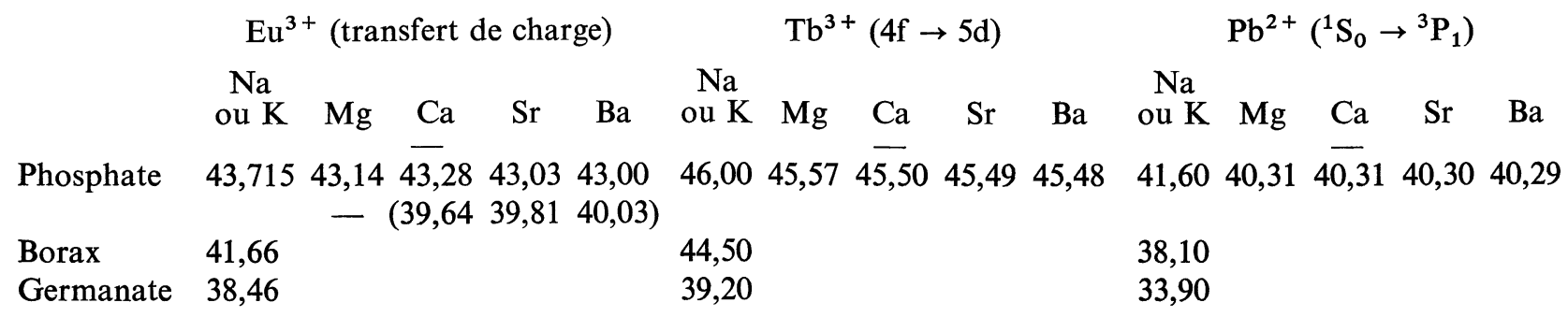

a montré que pour les ions $\mathrm{Eu}^{3+}$ (transfert de charge), $\mathrm{Tb}^{3+}(4 \mathrm{f} \rightarrow 5 \mathrm{~d})$ et $\mathrm{Pb}^{2+}\left({ }^{1} \mathrm{~S}_{0} \rightarrow{ }^{3} \mathrm{P}_{1}\right.$ associé à $6 s^{2} \rightarrow 6 s 6 p$ ) les maximums des spectres d'excitation se déplacent du côté des faibles énergies dans les séries respectives (Tableau IV) : $\mathrm{K}, \mathrm{Mg}, \mathrm{Ca}, \mathrm{Sr}, \mathrm{Ba}$, phosphate, borate, germanate.

Ceci rend possible le calcul de l'électronégativité optique $\chi_{\mathrm{op}}(X)$ par la relation de Jørgensen :

$$
\sigma_{\text {exp }}=30000\left[\chi_{\text {op }}(X)-\chi_{\text {(cation) }}\right]\left(\mathrm{cm}^{-1}\right)
$$

$\sigma_{\exp }$ : nombre d'onde du maximum de la bande d'excitation ou d'absorption ; $\chi_{\text {(eation) }}$ : électronégativité du cation central $\left(1,9\right.$ pour $\left.\mathrm{Eu}^{3+}\right)$.

De plus, le paramètre néphélauxétique $\beta$ peut être également déterminé par :

$$
\beta=\frac{\sigma_{1}-\sigma_{\mathrm{exp}}}{\sigma_{1}}
$$

$\sigma_{1}$ : nombre d'onde associé à la transition de l'ion libre.

Cet effet néphélauxétique est expliqué par Jørgensen comme une indication du degré de covalence de la liaison entre l'activateur et les ligandes. Il exprime la décroissance relative des paramètres électrostatiques de Racah de chaque configuration impliquée dans une transition. Les résultats de $\beta$ et $\chi_{\text {opt }}$ sont reportés sur la figure 11 où l'on voit que ces deux grandeurs ne sont pas influencées par le changement de $\mathbf{M}$ dans les séries MO- $\mathrm{P}_{2} \mathrm{O}_{5}$. Par contre la variation du réseau formateur entraîne des évolutions opposées de $\beta$ et $\chi_{\text {opt }}$. En effet, à une augmentation de la covalence entre $\mathrm{Eu}^{3+}$, $\mathrm{Tb}^{3+}$ ou $\mathrm{Pb}^{2+}$ et $\mathrm{O}^{2-}$ correspond bien une diminution de l'électronégativité du verre. Ces observations peuvent être reliées aux distances entre les ions du centre luminogène. Pour un même coordinat, l'abaissement des niveaux d'énergie correspond à une variation sensible, donc mesurable, de ces distances. Caro a pu mesurer ces écarts sur la position énergétique de ${ }^{2} \mathrm{P}_{1 / 2}$ du néodyme [25].

Des résultats intéressants ont aussi été obtenus par Reisfeld à partir de la transition $4 \mathrm{f} \rightarrow 5 \mathrm{~d}$ de l'ion $\mathrm{Ce}^{3+}[31-32]$. Le tableau $\mathrm{V}$ montre que le paramètre néphélauxétique $\beta$ croît dans la série : eau, phosphate, borate, silicate, ce qui exprime la tendance de la liaison covalente. Reisfeld en déduit que l'électronégativité des tétraèdres $\mathrm{BO}_{4}$ est plus petite que celle des tétraèdres $\mathrm{PO}_{4}$.

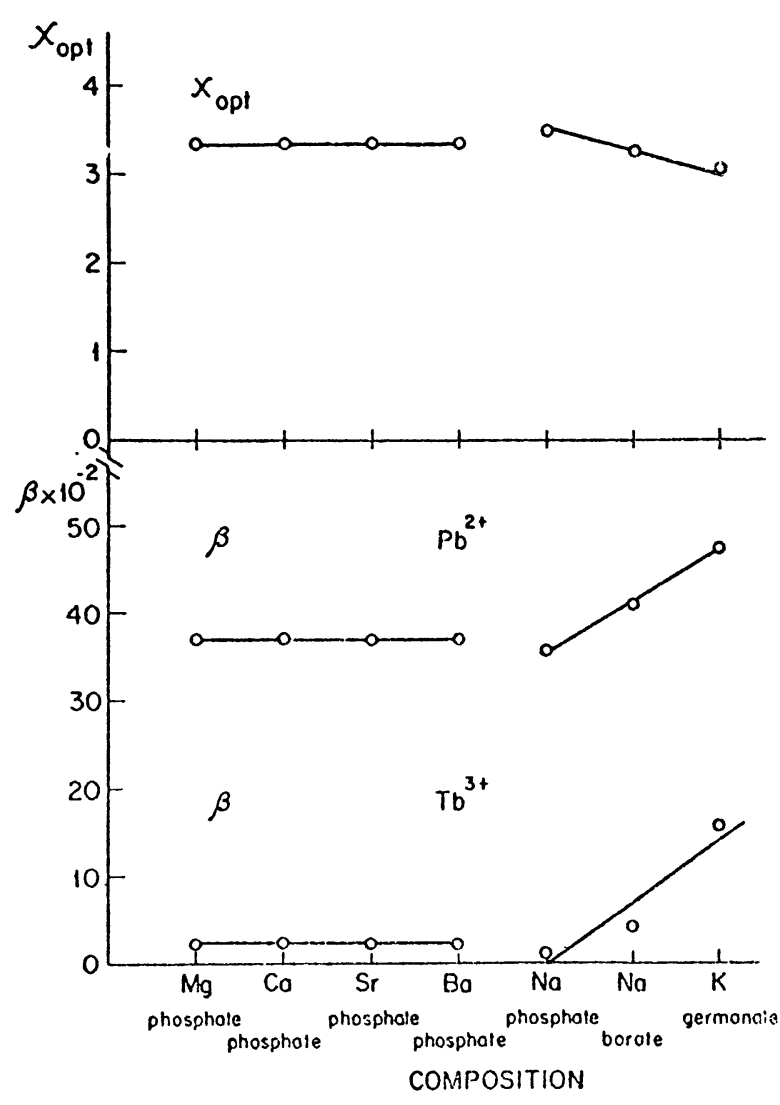

FIG. 11. - Variation de l'électronégativité optique $\chi_{\text {opt }}$ et du paramètre néphélauxétique $\beta$ dans les verres de métaphosphates MO- $\mathrm{P}_{2} \mathrm{O}_{5}(\mathrm{M}=\mathrm{Mg}, \mathrm{Ca}, \mathrm{Sr}, \mathrm{Ba}, \mathrm{Na})$, le borate de sodium et le germanate de potassium. D'après [28].

\section{TABLEAU V}

Energies des maximums $\sigma$ du spectre d'excitation de la bande $4 f \rightarrow 5 \mathrm{~d}$ de l'ion $\mathrm{Ce}^{3+}$ et valeurs du paramètre néphélauxétique $\beta$ de Jorgensen, d'après [31].

$\begin{array}{lccccc} & \text { Ion libre } & \text { Eau } & \begin{array}{c}\text { Phos- } \\ \text { phate }\end{array} & \text { Borate } & \text { Silicate } \\ \sigma \mathrm{cm}^{-1} & 51 \overline{0} & 39 \overline{600} & 33 \overline{333} & 3 \overline{-959} & 31500 \\ \beta & & 0,223 & 0,346 & 0,373 & 0,382\end{array}$

3. Caractérisation de la symétrie du site et évolution de la distorsion. - On a vu que la raie ${ }^{5} \mathrm{D}_{0} \rightarrow{ }^{7} \mathrm{~F}_{0}$ peut exister dans les sites de symétrie $C_{s}, C_{n}, C_{n v}$ qui 
permettent les processus dipolaires électriques. Dans les verres oxygénés, l'existence de cette transition, avec une intensité souvent forte, ainsi que la décomposition de la transition ${ }^{5} \mathrm{D}_{0} \rightarrow{ }^{7} \mathrm{~F}_{1}$ en trois composantes Starck sont en bon accord avec l'attribution de la basse symétrie ponctuelle du site au groupe $\mathrm{C}_{\mathrm{s}}$. Les résultats obtenus par Reisfeld sur les métaphosphates MO- $\mathrm{P}_{2} \mathrm{O}_{5}(\mathrm{M}=\mathrm{Na}, \mathrm{Mg}, \mathrm{Ca}, \mathrm{Sr}, \mathrm{Ba})$ sont reportés sur le tableau VI [28].

Le degré de distorsion peut être évalué par le rapport des intensités des transitions $\mathrm{DE}^{5} \mathrm{D}_{0} \rightarrow{ }^{7} \mathrm{~F}_{2}$, interdites dans l'ion libre, et des transitions $\mathrm{DM}{ }^{5} \mathrm{D}_{0} \rightarrow{ }^{7} \mathrm{~F}_{1}$. En normalisant l'intensité de ${ }^{5} \mathrm{D}_{0} \rightarrow{ }^{7} \mathrm{~F}_{1}$ à l'unité, celle relative à ${ }^{5} \mathrm{D}_{0} \rightarrow{ }^{7} \mathrm{~F}_{2}$ illustre immédiatement l'importance de la distorsion. Celle-ci diminue dans la série $\mathrm{Mg}, \mathrm{Ca}, \mathrm{Sr}, \mathrm{Ba}$ (Tableau VI). Comme l'électronégativité décroît dans cette même série, cela signifie que l'attraction est la plus forte entre $\mathbf{M g}^{2+}$ et les groupes $\mathrm{PO}_{4}$ donc que la distance moyenne entre le centre du cube occupé par $\mathrm{Mg}^{2+}\left(\mathrm{ou} \mathrm{Eu}{ }^{3+}\right.$ ) et les 8 oxygènes placés approximativement sur les sommets de ce cube distordu, est la plus grande. Il en résulte que la cavité cubique subit une distorsion plus importante dans les réseaux contenant du magnésium que dans ceux contenant du baryum [28]. Le champ cristallin sera donc plus élevé dans $\mathrm{BaO}-\mathrm{P}_{2} \mathrm{O}_{5}$ comme on peut s'en rendre compte sur la valeur des écarts des niveaux ${ }^{7} \mathrm{~F}_{1}$ (Tableau VI).

L'incorporation de $\mathrm{Ce}^{3+}$ dans les verres de borax et de phosphate apporte des informations à la fois qualitatives et quantitatives.

La transition $4 \mathrm{f} \rightarrow 5 \mathrm{~d}$ a déjà fait l'objet d'études approfondies. Les bandes d'absorption ultra-violettes traduisant les niveaux ${ }^{2} \mathrm{D}$ de $\mathrm{Ce}^{3+}$ peuvent contenir jusqu'à cinq composantes (dégénérescence de Kramer). On enregistre ces cinq composantes dans les borax et phosphate vitreux [31]. Il est donc possible de calculer les positions des centres de gravité des niveaux attachés aux orbitales $e_{g}$ et $t_{2 g}$ et, par voie de conséquence, de connaître le paramètre bien connu $10 \mathrm{Dq}$. Le tableau VII montre que $10 \mathrm{Dq}$ est pratiquement le même et que la distorsion interne aux deux matériaux vitreux est donc semblable.

3.4 CARACTÉRISATION DU NOMBRE DE SITES DANS LES MATÉRIAUX VITREUX. - Les légers déplacements des niveaux d'énergie dus aux différences d'environnements des ions activateurs provoquent une variation notable et évidemment un élargissement spectral parfois considérable. La comparaison des largeurs de bande à mihauteur dans les cristaux et les verres peut donner une indication sur le nombre de sites occupés. Par exemple,

\section{TABleAu VI}

Energies des transitions ${ }^{5} \mathrm{D}_{0} \rightarrow{ }^{7} \mathrm{~F}_{1},{ }^{6} \mathrm{D}_{0} \rightarrow{ }^{7} \mathrm{~F}_{0}$ en $\mathrm{cm}^{-1}$ et intensités de ${ }^{5} \mathrm{D}_{0} \rightarrow{ }^{7} \mathrm{~F}_{1}$ et ${ }^{5} \mathrm{D}_{0} \rightarrow{ }^{7} \mathrm{~F}_{2}$ de l'ion Eu ${ }^{3+}$ dans les métaphosphates $\mathrm{MO}-\mathrm{P}_{2} \mathrm{O}_{5}(\mathrm{M}=\mathrm{Na}, \mathrm{Mg}, \mathrm{Ca}, \mathrm{Sr}, \mathrm{Ba})$ d'après [28].

\begin{tabular}{|c|c|c|c|c|c|c|c|c|c|}
\hline & $\mathrm{Na}$ & & $\mathbf{M}$ & & $\mathrm{Ca}$ & & $\mathrm{Sr}$ & & $\mathrm{Ba}$ \\
\hline & $\sigma$ & $\Delta \sigma$ & $\sigma$ & $\Delta \sigma$ & $\sigma$ & $\Delta \sigma$ & $\sigma$ & $\Delta \sigma$ & \\
\hline & 16797 & & 17656 & & 16766 & & 16760 & & 16779 \\
\hline${ }^{5} \mathrm{D}_{0} \rightarrow{ }^{7} \mathrm{~F}_{1}$ & 16879 & & 16873 & 117 & 16886 & 120 & 16885 & 125 & \\
\hline & 16980 & 101 & 16990 & 117 & 17004 & 118 & 17004 & 119 & \\
\hline${ }^{5} \mathrm{D}_{0} \rightarrow{ }^{7} \mathrm{~F}_{0}$ & & & 17281 & & 17290 & & 17251 & & 17236 \\
\hline Intensité de ${ }^{5} \mathrm{D}_{0} \rightarrow{ }^{7} \mathrm{~F}_{1}$ & & & 1 & & 1 & & 1 & & 1 \\
\hline Intensité de ${ }^{5} \mathrm{D}_{0} \rightarrow{ }^{7} \mathrm{~F}_{2}$ & & & 7 & & 6,3 & & 5,7 & & 5,3 \\
\hline
\end{tabular}

TABLEAU VII

Maximum des spectres d'absorption de $\mathrm{Ce}^{3+}$ dans les verres. D'après [31]

Orbitales d

\begin{tabular}{|c|c|c|c|c|c|c|c|c|c|c|}
\hline & & \multicolumn{3}{|c|}{ Symétrie } \\
\hline & & $x^{2}-y^{2}$ & $2 z^{2}-x^{2}-y^{2}$ & $x y$ & $y^{2}$ & $x^{2}$ & $\mathrm{e}_{\mathrm{g}}$ & $t_{2 \mathrm{~g}}$ & sphérique & $10 \mathrm{D}_{Q}$ \\
\hline \multirow[b]{2}{*}{ Phosphate } & Nombre d'onde & 33500 & 40733 & 43956 & 47169 & 51282 & 36666 & 47000 & 42866 & 11444 \\
\hline & $\left\{\begin{array}{l}\text { Largeur de bande } \\
\text { en } \mathrm{cm}^{-1}\end{array}\right.$ & 816,8 & 1407,9 & 836,1 & 1133,7 & 986,55 & & & & \\
\hline \multirow[b]{2}{*}{ Borax } & $\begin{array}{l}\text { Nombre d'onde } \\
\mathrm{en} \mathrm{cm}^{-1}\end{array}$ & 31952 & 33454 & 39201 & 45125 & 47177 & 32706 & 43834 & 39383 & 11128 \\
\hline & $\begin{array}{l}\text { Largeur de bande } \\
\text { en } \mathrm{cm}^{-1}\end{array}$ & 2744 & 5184 & 6902 & 4572 & 3231 & & & & \\
\hline
\end{tabular}


la largeur de ${ }^{5} \mathrm{D}_{0} \rightarrow{ }^{7} \mathrm{~F}_{0}$ est environ de $2 \mathrm{~cm}^{-1}$ dans les cristaux et de $119 \mathrm{~cm}^{-1}$ dans le phosphate vitreux et $149 \mathrm{~cm}^{-1}$ dans le silicate vitreux [30]. Reisfeld en conclut qu'il y a cinquante sites légèrement différents de symétrie $\mathrm{C}_{\mathrm{s}}$ dans ces verres.

Nous pensons que cette évaluation n'est qu'approximative et qu'il est préférable d'utiliser la nouvelle technique d'affinement de raie de fluorescence ( $\mathrm{FLN}$ » ou « fluorescence line narrowing ») rendue exploitable par l'utilisation des lasers accordables dans tout le spectre visible.

La «FLN » fut d'abord mis en évidence dans le rubis par Szabo [41] puis dans les verres au néodyme par Riseberg [42] au moyen d'un laser à fréquence fixe. Il fallait qu'il existe des coïncidences entre l'énergie du photon excitateur et l'écart séparant deux niveaux d'un ion. La méthode fut ensuite étendue par Delsart et coll. [43] sur $\mathrm{LaAlO}_{3}\left(\mathrm{Pr}^{3+}\right)$ en utilisant un laser accordable. Ce n'est que depuis peu de temps qu'une étude systématique a été entreprise sur les verres dopés par $\mathrm{Eu}^{3+}, \mathrm{Yb}^{3+}$ et $\mathrm{Nd}^{3+}$ par Weber, Yen, Riseberg et Brecher [34-35-36]. Cette méthode présente l'avantage d'exciter sélectivement les sites individuels, d'atteindre leur largeur homogène et donc de s'affranchir des effets liés à l'élargissement inhomogène. Le laser accordable peut être continu ou pulsé. Dans ce dernier cas, il permet, en outre, la mesure du déclin de fluorescence donc le calcul de la probabilité d'émission radiative du niveau excité. Les performances dépendront de la largeur de la raie laser et de la résolution du monochromateur utilisé pour détecter la fluorescence.

Les résultats de "FLN" sur un verre de silicate dopé par $\mathrm{Yb}^{3+}$ sont montrés sur la figure 12. La trace

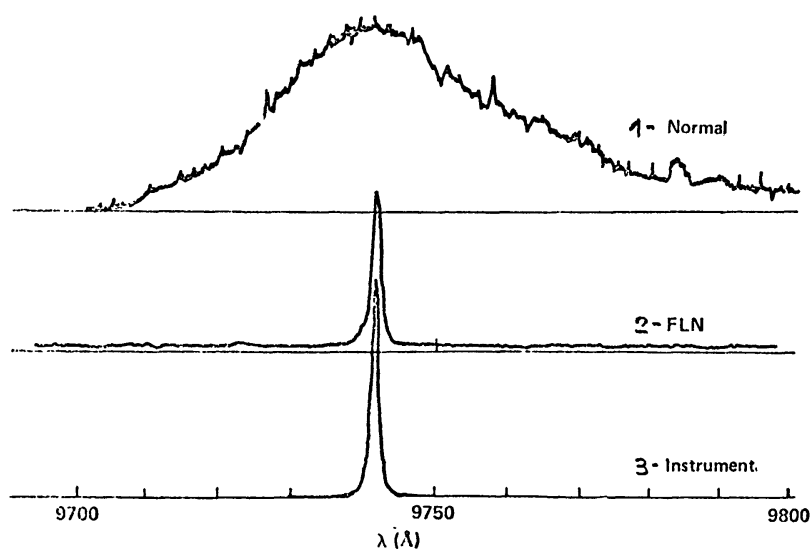

FIG. 12. - Affinement de raie de fluorescence (FLN) pour la transition ${ }^{2} \mathrm{~F}_{5 / 2} \rightarrow{ }^{2} \mathrm{~F}_{7 / 2}$ de $\mathrm{Yb}^{3+}$ dans des verres de silicates à $6 \mathrm{~K}$. L'échantillon contient 0,1 mole \% d'ytterbium. D'après [44].

supérieure (1) est le spectre de fluorescence inhomogène de la raie ${ }^{2} \mathrm{~F}_{5 / 2} \rightarrow{ }^{2} \mathrm{~F}_{7 / 2}$ observée sous excitation continue non sélective. Les deux autres traces montrent que la raie de fluorescence " FLN » (2) et la raie excitatrice résonante du laser (3) sont pratiquement comparables. Les largeurs de raie homogène de $\mathrm{Yb}^{3+}$ dans le silicate représentent les émissions optiques les plus étroites $\left(<0,5 \mathrm{~cm}^{-1}\right)$ reportées sur des matrices amorphes [44].

Les niveaux de $\mathrm{Eu}^{3+}$ se prêtent à deux types d'expériences : soit l'émission de fluorescence ${ }^{5} \mathrm{D}_{\mathrm{o}} \rightarrow{ }^{7} \mathrm{~F}_{\mathrm{o}}$ en résonance avec la raie excitatrice (Fig. 13-a) soit
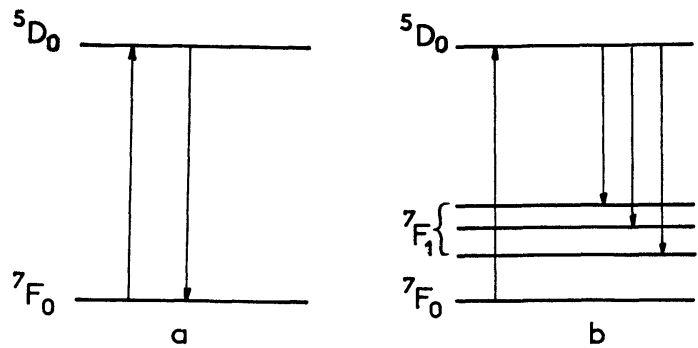

FIG. 13. $-a$ ) Raie de résonance $5 \mathrm{D}_{0} \rightarrow{ }^{7} \mathrm{~F}_{0}$ de l'ion $\mathrm{Eu}^{3+}$. b) Raies non résonantes ${ }^{5 D_{0}} \rightarrow{ }^{7} F_{1}$ de l'ion $\mathrm{Eu}^{3+}$ dans un verre de silicate. Pour les basses symétries, la décomposition de ${ }^{7} F_{1}$ est maximum. Sur ces schémas, la raie excitatrice du laser correspond à l'énergie de $7 \mathrm{~F}_{0} \rightarrow{ }^{5} \mathrm{D}_{0}$ d'un site.

l'émission de fluorescence ${ }^{5} \mathrm{D}_{\mathrm{O}} \rightarrow{ }^{7} \mathrm{~F}_{1}$ non résonante après excitation laser ${ }^{7} \mathrm{~F}_{\mathrm{O}} \rightarrow{ }^{5} \mathrm{D}_{\mathrm{o}}$ (Fig. 13b). Ce sont ces cas qui ont été particulièrement analysés par Weber (36) et Brecher (35) à la fois dans un silicate contenant $\mathrm{Na}, \mathrm{Ba}$ et $\mathrm{Zn}$ et dans le verre $\mathrm{Eu}\left(\mathrm{PO}_{3}\right)_{3}$. La largeur inhomogène de ${ }^{5} \mathrm{D}_{\mathrm{O}} \rightarrow{ }^{7} \mathrm{~F}_{\mathrm{o}}$ est voisine de $250 \mathrm{~cm}^{-1}$ et lorsque le laser est accordé sur cette bande, la décomposition des niveaux Starck varie de 150 à $550 \mathrm{~cm}^{-1}$. Weber a pu établir le diagramme de la figure 14 qui rend compte de la variation du champ

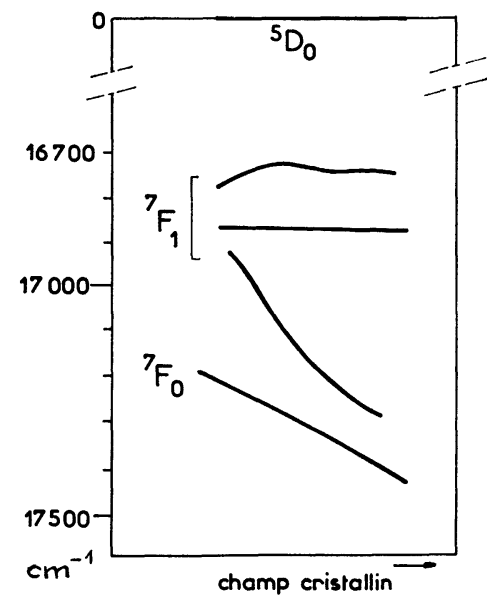

Fig. 14. - Schéma des niveaux d'énergie de ${ }^{5} D_{0},{ }^{7} F_{1}$ et ${ }^{7} F_{0}$ de l'ion $\mathrm{Eu}^{3+}$ dans le verre $\mathrm{Eu}\left(\mathrm{PO}_{3}\right)_{3}$ en fonction de l'intensité du champ cristallin. D'après [44]. Cette évolution est déduite de l'enregistrement des composantes de ${ }^{7} \mathrm{~F}_{1}$ selon la fréquence excitatrice ${ }^{7} \mathrm{~F}_{0} \rightarrow{ }^{5} \mathrm{D}_{0}$ dans la raie inhomogène du verre.

local des différents sites dans la matrice vitreuse. Le comportement de la structure de ces niveaux permet d'obtenir une connaissance détaillée de la distribution de la coordination des ions activateurs. Riseberg et Brecher ont calculé la variation des paramètres de 
champ cristallin $B_{q}^{k}$ en partant de l'hypothèse d'un site de symétrie $\mathrm{C}_{2 \mathrm{v}}$ avec une coordination 8 . Le déplacement d'un neuvième ion $\mathrm{O}^{2-}$ le long de l'axe de symétrie et la distorsion spatiale des prismes d'oxygènes qui en résulte permettent d'expliquer lesvariations du champ cristallin [35].

Enfin, en utilisant une raie excitatrice étroite (généralement inférieure à $2 \mathrm{~cm}^{-1}$ ), on peut comparer les largeurs de bande de « FLN » aux très basses tempéra- tures avec la largeur de raie inhomogène. Weber trouve $20 \mathrm{MHz}$ pour la transition ${ }^{5} \mathrm{D}_{\mathrm{O}} \rightarrow{ }^{7} \mathrm{~F}_{\mathrm{O}}$ dans un silicate à $1,7 \mathrm{~K}$ alors que la largeur inhomogène est environ $2000 \mathrm{GHz}$, si bien qu'il y a en réalité un rapport de l'ordre de $10^{5}$ entre ces deux valeurs. Le nombre de sites physiquement distinguables dans les verres s'avère donc beaucoup plus élevé que celui déterminé directement par comparaison entre les raies appartenant respectivement au cristal et au verre.

\section{Bibliographie}

[1] Bell, R. J., Dean, P. and Hilben-Buttler, D. C., J. Phys. C 32111 (1970); J. Phys. C 41214 (1971).

Bell, R. J., Rep. Progr. Phys. 35 (1972) 1315.

[2] Dean, P., Rev. Mod. Phys. 44 (1972) 127.

[3] Brawer, Phys. Rev. B 11 (1975) 3137.

[4] Lucovsky, G., Proc. 5th Int. Conf. liquid and amorphous semicond. Garmisch, 1099 (1974).

[5] Brodsky, M. H., Topics in applied physics " light scattering in solids » (Springer-Verlag-Berlin) ed. M. Cardona 8 (1975) 205.

[6] BötTgER, H., Phys. Stat. Sol. (b) 62 (1974) 9.

[7] Kittel, C., Introduction à la physique de l'état solide (Dunod) 1970.

[8] Di BARTolo, B., Optical interactions in solids (John Wiley and sons, inc. New-York) 1968.

[9] MoORAdian, A., Raman spectroscopy of solids laser handbook, (ed. by Arecchi and Schultz-Dubois), (NorthHolland) 1972 vol. 2, p. 1409.

[10] TURRell, G., Infrared and Raman spectra of crystals (Acad. press, London 1972.

[11] Poulet, H. et Mathieu, J. P., Spectres de vibration et symétrie des cristaux (Gordon et Breach, Paris) 1970.

[12] Born, M. et HuAng, K., Dynamical theory of crystal lattices (Clarendon press, Oxford) 1962.

[13] Larroque, D., Thèse de $3^{\mathrm{e}}$ cycle, Bordeaux I, 9 juillet 1976.

[14] Wilson, E. B., Decius, J. C., Cross, P. C., Molecular vibrations (Mc Graw-Hill, New-York) 1955.

[15] Couzi, M., Vignalou, J. R., Boulon, G., Solid State Commun. 20 (1976) 461.

[16] Shuker, R. and Gamon, R., Phys. Rev. Lett. 25 (1970) 222. Shuker, R. and Gamon, R., Proc. 2nd Int Conf. scattering in solids. Paris, (Flammarion-Paris) (1971) p. 334, M. Balkanski.

[17] Topics in applied physics-Light scattering in solids (ed. M. Cardona) (Springer Verlag, Berlin Heildelberg New-York) 8 (1975).

[18] Light scattering in solids-3d int. conf. Campinas (Brésil), M. Balkanski (Flammarion) 1975, Paris.

[19] Shuker, R. and Gamon, R., Phonons-Proc. int. conf. Rennes (N. A. Nusimovici) (Flammarion) 1971, p. 202, Paris.

[20] Finkman, E., Defonzo, A., Tauc, J., Proc. 5th int. conf. amorphous and liquid semiconductors-Garmisch (Taylor and Francis, London) (1973) p. 1275.
[21] Mooradian, A., Wright, G. B., Physics of selenium and tellurium (ed. W. C. Cooper), (Pergamon press Oxford) 1969 p. 169.

[22] BRIL, T. W. Raman spectroscopy of crystalline and vitreous borates. Thesis Eindhoven (Philips Research reports supplements) 2 (1976).

[23] CurIE, D., Luminescence cristalline (Dunod Paris) 1960.

[24] CurIE, D., Champ cristallin et luminescence, (GauthierVillars Paris) 1968.

[25] CARo, P., Structure électronique des éléments de transition, l'atome dans le cristal (Presses universitaires de France, Paris) 1976.

[26] Reisfeld, R., J. Res. Nat. Bur. Stand. 76A (1972) 613.

[27] Duffy, J. A. and Ingram, M. D., J. Chem. Phys. 52 (1970) 3752 : J. Inorg. Nucl. Chem. 36 (1974) 43.

[28] Reisfeld, R., Boehm, L., Ish-Slalom, M., Fischer, R., Phys. Chem. glass. 15 (1974) 76.

[29] Reisfeld, R. and Bozhm, L., J. Non-Crystall. Solids 16 (1974) 83.

[30] Reisfeld, R., Velapodi, R. A., Boehm, L. and Ish-ShaLOM, M., J. Phys. Chem. 75 (1971) 3980.

[31] Reisfeld, R., Hormodaly, J., Barnett, B., Chem. Phys. letters 17 (1972) 248.

[32] ReISFeLD, R., Struct. Bonding 13 (1973) 53.

[33] Reisfeld, R., Struct. Bonding, 22 (1975) 122.

[34] Weber, M. J., Paisner, J. A., Sussman, S. S., Yen, W. M. and Riseberg, L. A., Brecher, C., J. Luminescence $12 / 13$ (1976) 729.

[35] Brecher, C. and Riseberg, L. A., Phys. Rev. B 13 (1976) 81.

[36] Weber, W. J., Spectroscopie des éléments de transition et des éléments lourds dans les solides Lyon (1976). Colloque int. du C. N. R.S. (Mme Gaume) (à paraître).

[37] Jacquier, B. and Richardson, J. W., J. Chem. Phys. 63 (1975) 2442.

[38] JACQUier, B., J. Chem. Phys. 64 (1976) 4939.

[39] Moncorge, R., Jacquier, B., Boulon, G., GaumeMAHN, F., JANIN, J., J. luminescence $12 / 13$ (1976) 467.

[40] Valon, P., Gacon, J. C., Vedrine, A., Boulon, G., J. Solid State Chem. (à paraître 1976).

[41] SzaBo, A., Phys. Rev. Lett. 25 (1970) 924 and 27 (1971) 323.

[42] Riseberg, L. A., Phys. Rev. Lett. 28 (1972) 786 ; Solid State Commun. 11 (1972) 469 ; Phys. Rev. A 7 (1973) 671.

[43] Delsart, C., Pelletier-Allart, N. and Pelletier, R., Opt. Commun. 11 (1974) 84.

[44] Weber, W. J., Laser program. Annual report 1974, Lawrence livermore Laboratory UCRL, 50021 (1974). 